\title{
An acoustic-based approach for real-time deep-water navigation of an AUV
}

\author{
A. Tesei ${ }^{*}$, M. Micheli ${ }^{*}$, A. Vermeij ${ }^{*}$, G. Ferri ${ }^{*}$, M. Mazzi ${ }^{*}$, G. Grenon ${ }^{*}$, L. Morlando ${ }^{*}$ R. Costanzi ${ }^{* *}$, D. \\ Fenucci $^{* *}$, A. Caiti $^{* *} \&$ Andrea Munafö ${ }^{* * *}$ \\ * NATO Centre for Maritime Research and Experimentation La Spezia, ITALY \\ ** Dipartimento di Ingegneria dell'Informazione, Università di Pisa, Pisa, ITALY \\ *** Marine Autonomous \& Robotic Systems National Oceanographic Centre (NOC) Southampton, UK \\ * Corresponding Author. Email: alessandra.tesei@cmre.nato.int
}

\begin{abstract}
Synopsis
Navigation of Autonomous Underwater Vehicles (AUVs) remains a challenge due to the impossibility to use radio frequency signals and Global Positioning System (GPS). Navigation systems usually integrate different proprioceptive sensors to estimate the asset and the speed of the vehicle. In particular, the Doppler Velocity $\log$ (DVL) is fundamental during the navigation to have an accurate estimate of the vehicle's speed. This work addresses the enhancement of the navigation performance of an AUV through the development of a Deep Water Navigation Filter (DWNF). The DWNF is able to work in those scenarios where traditional navigation sensors show their limits: e.g., deep waters where DVL bottom lock cannot be achieved, or areas where the use of traditionally used static and dedicated beacons is incompatible with the mission requirements. The proposed approach exploits the concept of using a network of vehicles cooperatively supporting each other for their navigation. Several types of measurements coming from the different nodes (i.e. acoustic positioning system such as ship-mounted SSBL acoustic positioning system, USBL, range measurements from the different nodes) are fused in an Extended Kalman Filter framework with the odometry data. DWNF pushes forward the idea of using a network of robotic assets as a provider of navigation services allowing more flexible and robust operations of the deployed system.

The approach has been tested at sea during several experiments. We report here results from DWNF running successfully in real-time on the NATO STO-Centre for Maritime Research and Experimentation (CMRE) vehicles during the Dynamic Mongoose'17 experimentation off the South coast of Iceland (June-July 2017).
\end{abstract}

Keywords: Autonomous Underwater Vehicles; Deep Water navigation; Extended Kalman Filter; Data Fusion

\section{Introduction}

The navigation and localisation of Autonomous Underwater Vehicles (AUVs) in underwater remains challenging due to the combination of the attenuation of radio-frequency signals underwater making impossible the use of Global Positioning System (GPS) and of the low bandwidth and unreliability of typical underwater communications 0 .

Dead reckoning solutions which integrate several proprioceptive sensors are used to estimate through a navigation filter the position/attitude of the vehicle. Typically inertial sensors (accelerometers) are used in combination with gyroscopes to estimate the asset angle of the robot 0. Pressure sensors can provide reliable depth measurements 0 .

To compute the vehicle position the double integration of accelerations from accelerometers is usually avoided and a speed sensor is used. Commonly the used speed sensor is the Doppler Velocity Log 0 which provides an estimate of the vehicle speed relative to the seafloor. The best performance is obtained by using high grade Attitude and Heading Reference Systems (AHRS) 0. These systems include Fiber Optics Gyroscopes (FOGs) and a DVL and can achieve localisation errors as small as of the $0.1 \%$ of the travelled distance 0 .

To reduce the drift in the localisation error, different solutions are traditionally used. In Long-BaseLine (LBL) approaches some beacons (typically four) are moored around the operation area to provide range measurements to the vehicle, while in Ultra-Short Base-Line (USBL) the distance/bearing is measured with respect to a support vessel with known position 0 . LBL can provide a precise localisation, but the operation area is constrained by the region covered by the deployed beacons. With USBL, instead, a support vessel has to follow the AUV during the mission to transmit the position updates. These acoustic corrections are needed above all if the DVL cannot track the seafloor due to the excessive vehicle altitude (> $200 \mathrm{~m}$, but depends on the DVL models). In that case it

\footnotetext{
Author's Biography

Alessandra Tesei is a Senior Scientist at NATO STO Centre for Maritime Research and Experimentation (CMRE) in La Spezia, Italy. Her background is in underwater acoustics and sonar, acoustic signal processing, structural and physical acoustics, maritime autonomous vehicles, navigation. At the present she is leading the "Maritime Unmanned Systems for ASW" project at CMRE.
} 
can only estimate the AUV speed relative to the water incurring in potentially large drifts in the position estimate. This condition is referred as "deep water" navigation.

The presented solutions are today being complemented by dynamic robot networks in which the robotic nodes use cooperative localisation approaches 00 . The nodes can use underwater communication to improve their localisation estimate thanks to range/bearing measurements obtained periodically from the other assets.

These kinds of approaches aim at improving the team localisation performance without the need of dedicated vessels or deployed beacons. Cooperative localisation approaches are also beneficial when robots with different navigation capabilities are present in the network. In this case, the robots equipped with accurate sensors can support the navigation of vehicles endowed with less accurate localisation capabilities 0 .

The work presented in this paper pushes in this direction. We describe a Deep Water Navigation Filter (DWNF) to enhance the navigation performance of an AUV. DWNF is able to work in those scenarios where traditional approaches show their limits: e.g., deep waters where DVL bottom lock cannot be achieved, or areas where the use of static and dedicated beacons is incompatible with the mission requirements.

The filter fuses data from its proprioceptive sensors, (the Inertial Measurement Unit - IMU to estimate the asset angles, a pressure sensor to compute the depth and the DVL for the measurement of the speed - relative to the water in the case of absence of bottom lock) in an Extended Kalman Filter (EKF) framework using a kinematic model of the AUV. The AUV is considered as a mobile asset in an underwater, hybrid surveillance network 0 composed of mobile (either underwater or surface, either manned or unmanned) nodes and static nodes (gateway buoys). Through acoustic communications, the information is shared among the different nodes producing additional measurements relative to the other assets. In the addressed system the AUV can measure relative direction (through USBL) and relative distance (round trip time - RTT - by means of underwater modems) with respect to the other network nodes. Moreover the Kongsberg high-accuracy acoustic positioning system $\mathrm{HiPAP}^{\mathrm{TM}} 501$ is hull-mounted on the support ship to measure the position of the mobile assets.

The proposed approach extends the work in 0 and 0 and was designed taking into account the challenges of the addressed scenario. The system can simultaneously estimate the vehicle position and the sea current to provide more reliable predictions when measurements are not present for prolonged time. An outlier detection and rejection strategy based on the Mahalanobis distance associated to each measurement is implemented. The filter takes into account the problems related to acoustic communications, such as the delays introduced by the Time Division Multiple Access (TDMA) Medium Access Control protocol used in the acoustic network. In order to handle the delays between the instant of measurement and the availability of the data, and to exploit also measurements that are older than the ones already available due to possible communication delays, the developed DWNF maintains a memory of the past estimations, inputs and measurements with associated timestamps. As soon as a new observation is available, it is applied in the right instant of the past, then all the following inputs and measurements in the database are re-applied in order to update the following estimates.

The cooperative navigation system has been tested at sea in realistic conditions. We report results of the DWNF running on the NATO STO-Centre for Maritime Research and Experimentation (CMRE) Ocean Explorer (OEX)

- C AUVs conducted at sea during a NATO operational exercise, the Dynamic Mongoose'17, off the South coast of Iceland (June-July 2017). Achieved results show the robustness of the system and demonstrate how an underwater acoustic network can offer navigation services for its nodes at the cost of a minimal impact on the normal network operation.

\footnotetext{
Author's Biography

Alessandra Tesei is a Senior Scientist at NATO STO Centre for Maritime Research and Experimentation (CMRE) in La Spezia, Italy. Her background is in underwater acoustics and sonar, acoustic signal processing, structural and physical acoustics, maritime autonomous vehicles, navigation. At the present she is leading the "Maritime Unmanned Systems for ASW" project at CMRE.
} 


\section{The Deep Water Navigation Filter}

The DWNF uses a kinematic model of the AUV for the prediction step. The model considers only the horizontal plane. Depth is reliably measured by the on board pressure sensor. The used model is reported in (1).

$$
\begin{aligned}
& x_{k+1}=x_{k}+\Delta t\left(\tilde{s}_{o w, k} \cos \tilde{\theta}_{o w, k}+u_{k}\right) \\
& y_{k+1}=y_{k}+\Delta t\left(\tilde{s}_{o w, k} \sin \tilde{\theta}_{o w, k}+v_{k}\right) \\
& u_{k+1}=u_{k}+\Delta t v_{u} \\
& v_{k+1}=v_{k}+\Delta t v_{v}
\end{aligned}
$$

The filter state, further than horizontal position components along the North and the East directions $\left[\begin{array}{ll}x_{k} & y_{k}\end{array}\right]^{T}$, includes the components of the sea current velocity along same directions $\left[\begin{array}{ll}u_{k} & v_{k}\end{array}\right]^{T}$. The water current velocity components are assumed constant (uniform current model, according to 0), except for a white Gaussian noise representing the model uncertainties along the two directions $\left(v_{u} \sim N\left(0, Q_{u}\right)\right.$ and $\left.v_{v} \sim N\left(0, Q_{v}\right)\right)$. In this way, the water current components can be estimated when absolute measurements are received. A good water current estimate can improve the filter prediction step, even in the case of correction lack for a certain period (because of the nature of the acoustic channel, this may happen despite the use of heterogeneous sources of information and complementary sensors). The inputs of the model are the speed and the course over water (model inputs $\left[s_{o w, k} \theta_{o w, k}\right]^{T}$ with $k$ the discrete time index) provided by the AUV front-seat, exploiting measurements from DVL with no bottom-lock along with the OEX-C heading estimates. The input is a noisy signal modelled as the sum of the actual value of the measured quantity and a zero-mean Gaussian noise.

$\tilde{s}_{o w, k}=s_{o w, k}+v_{s}$

$\tilde{\theta}_{o w, k}=\theta_{o w, k}+v_{\theta}$

with $v_{s} \sim N\left(0, Q_{S}\right)$ and $v_{\theta} \sim N\left(0, Q_{\theta}\right)$.

Different observation models are used for the external measurements coming from the other network nodes.

\section{1) $U S B L$}

USBL (bearing with respect to another node) - the acoustic modems used for communication within the acoustic network have USBL capabilities. The device is therefore capable to compute a measurement of the direction of arrival (DOA) once it receives a message. This is an observation of the navigation state that is used for the update step of the filter according to the model:

$z_{U S B L}=\operatorname{atan} 2\left(\tilde{y}_{i, k}-y_{k}, \tilde{x}_{i, k}-x_{i, k}\right)+\eta_{U S B L}$

that is a function of the position $\left[\begin{array}{ll}x_{i, k} & y_{i, k}\end{array}\right]^{T}$ of the remote asset that sent the message. atan 2 is the four-quadrant inverse tangent function and $\eta_{U S B L}$ models the measurement noise. The position of the remote asset is modelled as a random variable defined as the sum of the actual position and an additive zero-mean additive noise. It is estimated in parallel on-board through dedicated filters that exploit information, shared through the network, about the position of the various assets.

2) High-accuracy ship-mounted SSBL acoustic positioning system (Kongsberg HiPAP $P^{T M}$ 501) HiPAP $^{\mathrm{TM}}$ (provides $\mathrm{x}-\mathrm{y}$ position) - the high-accuracy positioning system is integrated on the support vessel. Relative position measurements are converted in absolute position with respect to the local reference NED frame through the integration with the navigation system (position and heading) of the vessel. NED measurements of the AUVs absolute position are communicated by exploiting the acoustic network and they are used for the estimation correction through the model:

$\mathbf{z}_{H i P A P}=\left[\begin{array}{l}x_{k} \\ y_{k}\end{array}\right]+\boldsymbol{\eta}_{H i P A P}$

where an additive zero-mean Gaussian noise $\boldsymbol{\eta}_{\text {HiPAP }}$ is considered. In case of surface navigation (e.g. after deployment before mission starting) with GPS access, after conversion from Latitude and Longitude coordinates to the local NED frame, an analogous model can be used.

3) Network-produced range 
Network-produced range (range measurements to other nodes) - using a navigational layer implemented at the highest level of the network 0 , timestamps enclosed in the exchanged messages are used to compute the Round Trip Time (RTT). RTTs, through a local indirect measurement of the speed of sound, allow to compute the distance of the AUV with respect to the remote asset. The computed range is compensated for the depth difference between the two involved nodes (the AUV and the remote asset) and is used as a navigation state observation according to the following model:

$$
z_{r n g}=\sqrt{\left(\tilde{x}_{i, k}-x_{k}\right)^{2}+\left(\tilde{y}_{i, k}-y_{k}\right)^{2}}+\eta_{r n g}
$$

where an additive zero-mean Gaussian measurement noise $\eta_{r n g}$ has been assumed. Considerations about the position of the remote asset reported for USBL DOA measurements apply also in the case of network-produced ranges. This approach does not require the addition of dedicated specific hardware and is offered by the network as an additional localisation service at the cost of occupying some bandwidth of the communication channel (see 0 for details).

The proposed DWNF is specifically designed to handle the typical issues related to the acoustic-based measurements, such as time irregularity, latency and presence of outliers 0 . A policy of outlier detection and rejection is implemented. The Mahalanobis distance associated to each measurement is calculated. The resulting value is compared with thresholds to decide whether to accept or discard the measurement to feed the filter. Thresholds are derived for each sensor from an extensive analysis of historical data collected during recent years for the involved sensors in NATO STO CMRE experimental activities 0.

The external measurements are shared through the acoustic communication channel. This creates delay in the reception of the measurements, due to the particular means access policy used (Time Division Multiple Access TDMA in this case) and to the non-negligible propagation time. To handle this, the DWNF maintains a memory of the past estimations, inputs and measurements with associated timestamps. A database is associated with each measurement type. The database maintains the various entries ordered by associated timestamp. When a new measurement with an associated timestamp older than the latest computed estimate is available, it is added in the right position and the history present in the database is used. Specifically, all the estimates more recent than the measurement timestamp are deleted. The most recent remaining estimate is used to apply immediately all the subsequent measurements and inputs through standard prediction and update steps of the EKF paradigm. In this way the new information is taken correctly into account to produce the last estimate of the filter 0 . The same solution is adopted in the case of availability of delayed inputs.

Further than the EKF running on the AUV with the objective of estimating its own navigation state, other EKF instances, customized on the basis of available sensors, are allocated either on the AUV itself or on the other network mobile nodes in order to filter or smooth local measurements. A key role is played by the filters implemented on board the support vessel.

Here, a filter is implemented for each asset with on-board a HiPAP ${ }^{\mathrm{TM}}$ transponder. The main goal is to estimate the position of these assets, but other useful benefits can be obtained. Since these filters are not executed on the vehicle of which the navigation state is estimated, they do not have direct access to the variables that may be considered the process inputs. The model described at the beginning of this section is, thus, not suitable for the purpose. The model of an autonomous (no input) system is used for the prediction step of the filter. The purely kinematic model includes, among the state components, the position $\left[\begin{array}{ll}x_{k} & y_{k}\end{array}\right]^{T}$ and the velocity $\left[\begin{array}{ll}\dot{x}_{k} & \dot{y}_{k}\end{array}\right]^{T}$ of the asset in the North-East reference frame:

$x_{k+1}=x_{k}+\Delta t \dot{x}_{k}$
$y_{k+1}=y_{k}+\Delta t \dot{y}_{k}$
$\dot{x}_{k+1}=\dot{x}_{k}+\Delta t v_{\dot{x}}$
$\dot{y}_{k+1}=\dot{y}_{k}+\Delta t v_{\dot{y}}$

The process noise $\left[v_{\dot{x}} v_{\dot{y}}\right]^{T}$, affecting only the velocity components, models the uncertainty on the asset dynamics. Through the ship-mounted SSBL observations, the state estimation is corrected. The measurement model for these filters is the same used for the filters on the AUVs.

Thanks to frequent HiPAP ${ }^{\mathrm{TM}}$ measurements (the sensor, although based on acoustics, does not interfere with the communication as it operates at different frequencies) a reliable estimation of the AUV motion is calculated on the support vessel for mission monitoring purposes. The resulting estimation is a smoothing of the HiPAP ${ }^{\mathrm{TM}}$ measurements (smoother EKF). This approach offers an immediate added value. The outlier detection and rejection algorithm based on Mahalanobis distance is applied also for these filters. This allows to identify potential outliers before injecting them in the network implying the double benefit of avoiding misleading measurements to arrive at the AUVs and not to occupy network bandwidth with bad information. 
A filter of the same kind is used on-board the support ship to estimate the position of the USBL modem mounted on a towed depressor. The ship communicates with the network thanks to this device that is towed at a distance of about hundred meters. An accurate estimate of USBL position is of fundamental importance for the DWNF to work properly as the measurement models depend on it. In order to minimise the potential error due to wrong assumptions on the ship USBL position, a HiPAP ${ }^{\mathrm{TM}}$ transponder is mounted attached to the depressor itself. The ship USBL position that is shared on the network to support the navigation of the AUVs is the one estimated this way.

\section{Selection of experimental results}

We report results from tests of the system during a NATO operational exercise, the Dynamic Mongoose'17 (DMON17) experimentation, conducted off the South coast of Iceland (26 June - 6 July 2017). In DMON17, CMRE's Cooperative ASW programme 0 aimed to deploy a robotic networked multi-static sonar network 0 into an operationally relevant environment. The CMRE multi-static network is usually deployed in littorals where bottom lock is available. In DMON17 the network was deployed in a region where deep waters areas were present. The DWNF filter was running in real-time on the OEX-C AUVs and the network was configured to share the acoustic measurements to be used for the cooperative navigation. Exploring the possibility to use the described system as a reliable navigation system for deep waters area was one of the trial's objectives.

Several missions were carried out, each one involving a different configuration of the CMRE network. The most remarkable achievement during the experiment was that the OEX-C AUVs exploited the described DWNF to navigate in a deep water area without the necessity of GPS position fix for an entire day of operations.

The OEX on-board software architecture is based on the Frontseat/Backseat paradigm and is based on the MOOS robotics software middleware 0 The navigation functionalities of the AUV are executed by a iXblue PHINS integrated with the frontseat computer. It filters the raw signal of the sensors to compute a navigation state estimation. The proposed DWNF is implemented on the backseat computer. Proprioceptive measurements are periodically $(1 \mathrm{~Hz})$ communicated through the vehicle bus by the frontseat computer. Exteroceptive measurements are available through the acoustic network by means of the USBL modem. The estimations computed by DWNF on the backseat are communicated to the frontseat, that exploits them to refine the navigation state as they were acoustic position fixes (i.e. LBL, USBL).

The DWNF was fully integrated in the control loop of the two CMRE OEX-C vehicles during all the different missions conducted in deep water configuration mode along the whole experimentation (up to about $11 \mathrm{~h}$ continuously).

Several heterogeneous instances of the filter were simultaneously active, both on the OEX-C itself for the position estimation of the other assets and on-board the top side (on the support vessel) for an upstream rejection of the HiPAP ${ }^{\mathrm{TM}}$ outliers. No integration issues emerged during their functioning.

Among all the missions conducted along DMON'17 experimentation, the mission conducted by Groucho, one of the two OEX-C AUV's operating during the exercise, on July $5^{\text {th }}, 2017$, starting at 9:00 GMT and lasting 15795s $\left(>4 \mathrm{~h}\right.$ ) has been selected. In this mission, a lack of $\mathrm{HiPAP}^{\mathrm{TM}}$ measurements was simulated in real-time, by imposing communication inhibition (up to $\sim 1 \mathrm{~h}$ ) towards the AUV for some parts of the path (see the HiPAP ${ }^{\mathrm{TM}}$ data received on-board the AUV with respect to those available on NRV Alliance along the full mission, as represented on the North-East plane in Figure $1(\mathrm{a})$ ). We underline that HiPAP ${ }^{\mathrm{TM}}$ measurements are the most informative for the AUV localisation since they are position fixes. In the described scenario the use of the $\mathrm{HiPAP}^{\mathrm{TM}}$ forced the support ship to be constrained in its navigation to stay close to the vehicles (up to a maximum of $2 \mathrm{~km}$ ). This is undesirable. For this reason in the experiments we sometimes inhibited the use of measurements from $\mathrm{HiPAP}^{\mathrm{TM}}$ to evaluate the navigation performance in the condition in which no vessel could be dedicated to support the vehicle navigation. It has to be remarked that USBL systems can be mounted on an Autonomous Surface Vehicle (ASV) to provide position fixes to the AUV, providing position fixes to the AUVs without the need of a dedicated ship. 


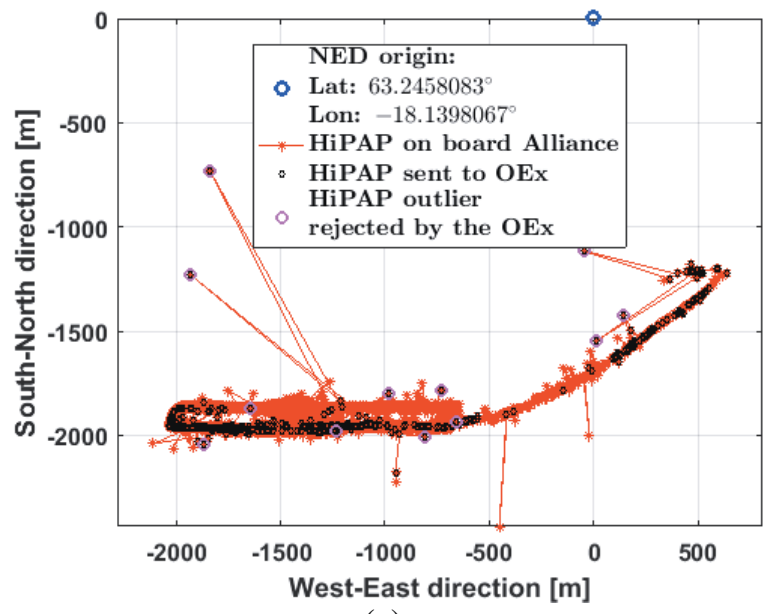

(a)

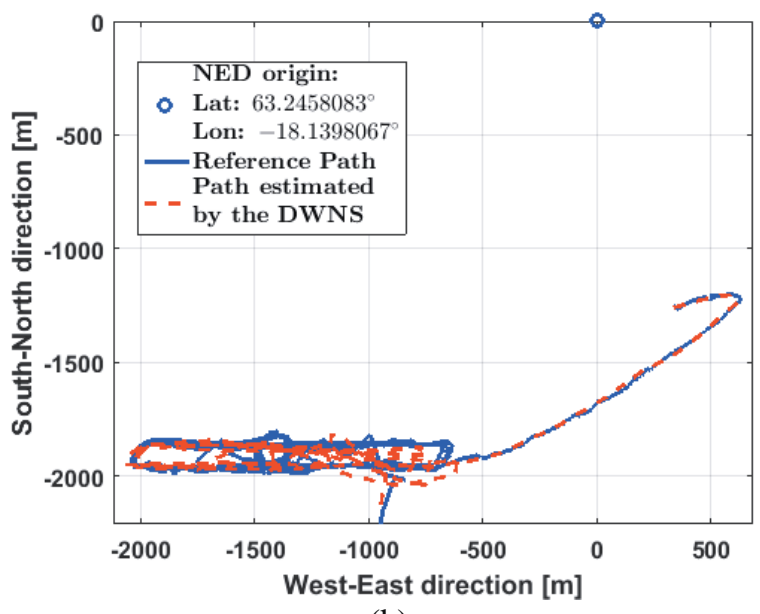

(b)

Figure 1: (a) HiPAP ${ }^{\mathrm{TM}}$ measurements on Alliance and on Groucho OEX-C AUV, and identified outliers; (b) Estimated path compared with all the position measurements (GPS and HiPAP ${ }^{\mathrm{TM}}$ ) available, which contribute to the Reference Path (RP). The reference system selected is a Local Tangent Plane NED, the origin of which is indicated in the legendas

The trial was conducted in an area where the bottom lock was available (about 130m of depth); however the velocity over ground was not integrated in the DWNF, which exploited only the velocity over water measurements.

In order to evaluate the behaviour of the DWNF as reference path (RP), the DVL output based on the speed over ground is considered.

In the selected mission, in addition to Groucho OEX two assets were involved: the support vessel (NRV Alliance) and a static node (a gateway buoy). The mission lasted more than 4 hours for a total travelled distance of about 16 kilometres at 80 metres of depth. Figure 1(b) shows the estimated positions compared to the RP. From the figure, it can be noted that the position accuracy is degraded when HiPAP ${ }^{\mathrm{TM}}$ data are unavailable. The filter, however, during these periods uses USBL and range RTT range measurements to limit the dead reckoning error. The vehicle is thus always provided with a reliable navigation state estimation, as shown in the plot of the position error in Fig. 2(a). The HiPAP ${ }^{\mathrm{TM}}$ measurements at the corresponding time on NRV Alliance (red stars) and on-board the OEX Groucho (black stars) are also reported in the lower part of the plot of Fig. 2(a).

It is interesting to notice in Fig. 2(b) the occurrence of data coming from USBL, RTT (and GPS) with respect to HiPAP $^{\mathrm{TM}}$ and how they support the EKF to provide estimates when the ship acoustic positioning system is lacking. Given the sparsity of HiPAP ${ }^{\mathrm{TM}}$ data availability on board the AUV, the choice on an opportunistic method is clearly justified and results effective.

Fig 3 shows the sea current intensity and direction estimated during the mission. A ground truth is not available to validate the estimation capabilities.

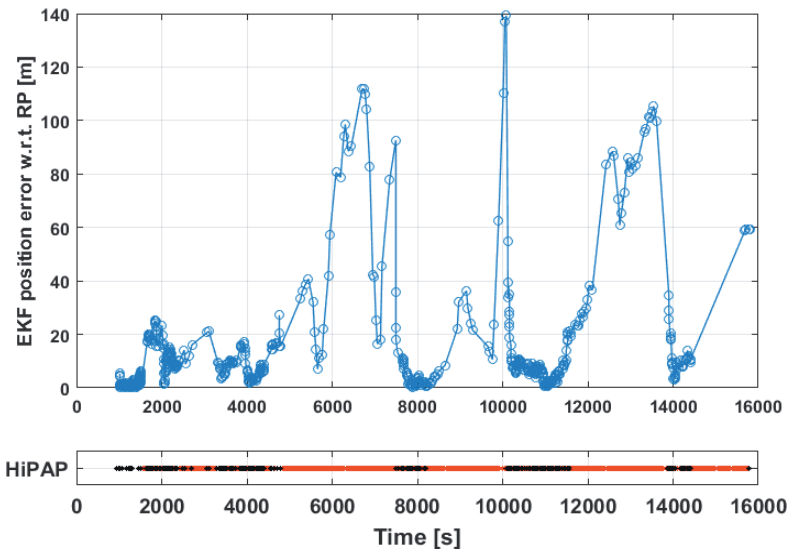

(a)

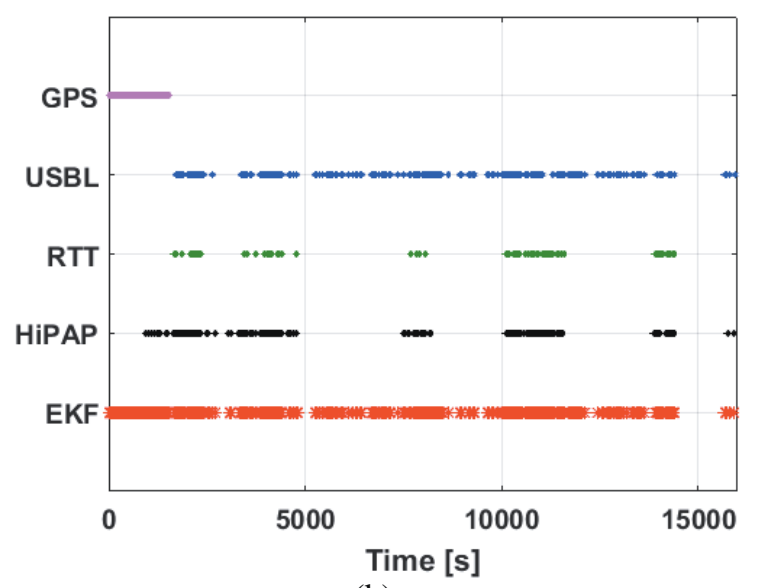

(b)

Figure 2: (a) EKF Position error along time. (b) Occurrences of measurements and estimations on Groucho AUV 

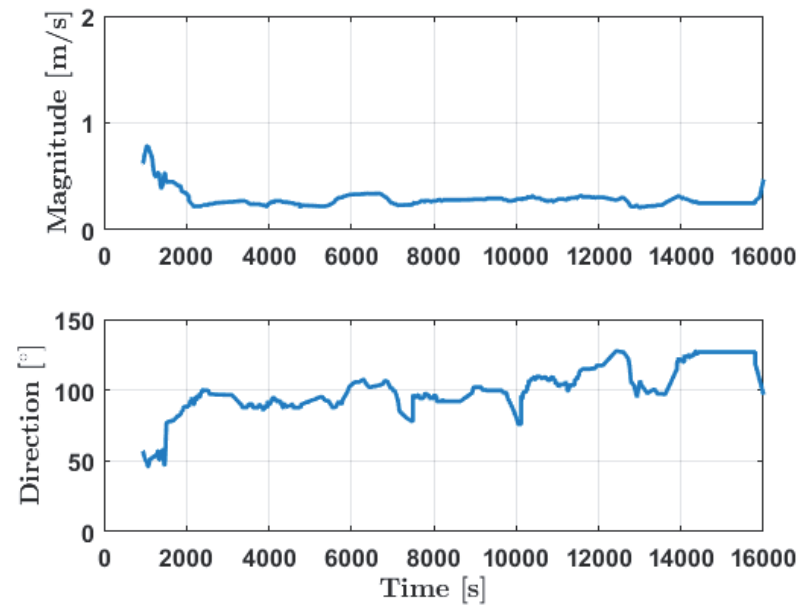

Figure 3: Estimated sea current speed (in magnitude and direction). Unfortunately no ground-truth data were available along the sea trials

\section{Acknowledgements}

The authors are grateful to all CMRE personnel involved in the preparation and execution of DMON17 exercise for their dedication and high professional skills, as well as to ITAN CO, XO and crew of NRV Alliance for their enthusiastic and continuous support during the trials. This work was funded by NATO Allied Command for Transformation under the Project "Maritime Unmanned Systems for ASW" of the CMRE Programme of Work.

\section{References}

Antonelli G.: "Underwater Robots", Springer Tracts in Advanced Robotics, 2014.

Benjamin, M., Newman P., Schmidt H. \& Leonard J.: "An overview of MOOS-IvP and a users guide to the IvP Helm autonomy software”, MIT, Cambridge, MA, USA, Tech, Report, MIT-CSAIL-TR-2010-041, 2010.

Costanzi R., Fenucci D., Giagnoni S., Munafò A. \& Caiti A.: "An Evaluation of Deep Water Navigation Systems for Autonomous Underwater Vehicles." IFAC-PapersOnLine 50.1, pp. 13680-13685, 2017.

Ferri G., Munafò A., Tesei A., Braca P., Meyer F., Pelekanakis K., Petroccia R., Alves J., Strode C. \& Lepage K.: "Cooperative Robotic Networks for Underwater Surveillance: an Overview", IET Radar, Sonar \& Navigation 11(12), July 2017.

Ferri G., Munafò A., LePage K.D.: "An Autonomous Underwater Vehicle Data-Driven Control Strategy for Target Tracking", IEEE Journal of Oceanic Engineering, Vol. 43, No2, April 2018.

Hegrenæs Ø., Ramstad A., Pedersen T. \& Velasco D.: "Validation of a new generation DVL for underwater vehicle navigation". Autonomous Underwater Vehicles (AUV), IEEE/OES, pp. 342-348, 2016.

Hodge V. \& Austin J.: "A survey of outlier detection methodologies." Artificial intelligence review, vol. 22 (2), pp. 85-126, 2004.

LePage K.D., Goldhahn R., Alves J., Strode C., Braca P., Ferri G., ... \& Biagini S.: "Autonomous Networked Anti-Submarine Warfare Research and Dvelopment at CMRE", Proceedings of MTS/IEEE OCEANS 2015, Genova (Italy), May 2015.

Maczka D.K., Gadre A.S. \& Stilwell D.J.: "Implementation of a cooperative navigation algorithm on a platoon of Autonomous Underwater Vehicles," Proceedings of MTS/IEEE OCEANS'07, Vancouver, BC, Canada, Sept. 2007.

Munafò A. \& Ferri G., "An acoustic network navigation system.” Journal of Field Robotics, vol. 34 (7), pp. 1332-1351, 2017.

Paull L., Saeedi S., \& Li H., "AUV Navigation and Localization: A Review", IEEE Journal of Oceanic Engineering. Vol. 39 (1), pp. 131-149, Jan. 2014. 